\title{
On the numerical solutions of
} open arc

\author{
Hu Li ${ }^{1 *}$ (D) Jin Huang ${ }^{2}$ and Guang Zeng ${ }^{3}$
}

\author{
"Correspondence: \\ lihu_0826@163.com \\ 'school of Mathematics, Chengdu \\ Normal University, Chengdu \\ 611130, China \\ Full list of author information is \\ available at the end of the article
}

\begin{abstract}
For the scattering problems of acoustic wave for an open arc in two dimensions, we give a uniqueness and existence analysis via the single layer potential approach leading to a system of integral equations that contains a weakly singular operator. For its numerical solutions, we describe an $O\left(h^{3}\right)$ order quadrature method based on the specific integral formula including convergence and stability analysis. Moreover, the asymptotic expansion of errors with odd power $O\left(h^{3}\right)$ is got and the Richardson extrapolation algorithm (EA) is used to improve the accuracy of numerical solutions. The efficiency of the method is illustrated by a numerical example.
\end{abstract}

Keywords: Quadrature method; Extrapolation algorithm; Scattering problems; Smooth open arc

\section{Introduction}

The scattering problems of electromagnetic waves or time-harmonic acoustic waves by an infinitely long semi-cylindrical obstacle with a smooth open contour cross-section $\Gamma \subset R^{2}$ are reduced to the Helmholtz equation with unbounded boundary value problems

$$
\left\{\begin{array}{l}
\Delta u(x)+k^{2} u(x)=0, \quad x \in R^{2} \backslash \Gamma, \\
u(x)=g(x), \quad x \in \Gamma, \\
\frac{\partial u(x)}{\partial r}-i k u(x)=o\left(\frac{1}{\sqrt{r}}\right), \quad r=|x| \longrightarrow \infty,
\end{array}\right.
$$

where $\Gamma$ is a smooth open arc, $k>0$ is the wave number, and $|x|$ is the Euclidean distance. The solution of (1) is got by the single layer potential theory in the following form [1]:

$$
u(y)=\int_{\Gamma} K_{0}(|x-y|) v(x) d s_{x}, \quad y \in R^{2} \backslash \Gamma,
$$

where $K_{0}|x-y|$ is the fundamental solution of (1), which is described as

$$
K_{0}(|x-y|)=\frac{i}{4} H_{0}^{(1)}(k|x-y|), \quad x \neq y
$$

and $H_{0}^{(1)}=i N_{0}+J_{0}$ is the Hankel function of order zero and of the first kind.

(c) The Author(s) 2020. This article is licensed under a Creative Commons Attribution 4.0 International License, which permits use, sharing, adaptation, distribution and reproduction in any medium or format, as long as you give appropriate credit to the original author(s) and the source, provide a link to the Creative Commons licence, and indicate if changes were made. The images or other third party material in this article are included in the article's Creative Commons licence, unless indicated otherwise in a credit line to the material. If material is not included in the article's Creative Commons licence and your intended use is not permitted by statutory regulation or exceeds the permitted use, you will need to obtain permission directly from the copyright holder. To view a copy of this licence, visit http://creativecommons.org/licenses/by/4.0/. 
$N_{0}$ is the Neumann function of order zero, which is given by

$$
N_{0}(z)=\frac{2}{\pi}\left(\ln \frac{z}{2}+\gamma\right) J_{0}(z)+\frac{2}{\pi} \sum_{n=1}^{\infty}\left(\sum_{m=1}^{n} \frac{1}{m}\right) \frac{(-1)^{(n+1)}}{(n !)^{2}}\left(\frac{z}{2}\right)^{2 n}
$$

with $\gamma=0.57721 \ldots$ denoting the Euler constant, and $J_{0}$ is the Bessel function of order zero, which is given by

$$
J_{0}(z)=\sum_{n=0}^{\infty} \frac{(-1)^{n}}{(n !)^{2}}\left(\frac{z}{2}\right)^{2 n}
$$

Equations (4) and (5) are valid for small $|z|$. For large $|z|$, they are inaccurate, and other series expressions are given in [2].

We decompose the integral kernel to analyze its properties as follows:

$$
K_{0}(|x-y|)=k_{1}(x, y)+k_{2}(x, y)
$$

where $k_{1}(x, y)=-1 / 2 \pi \ln |x-y|$ is a logarithmic singular function and $k_{2}(x, y)=i / 4-$ $1 / 2 \pi(\ln (k / 2)+\gamma)+O(|x-y| \ln |x-y|)$ is a smooth function.

The singe layer potential (2) solves the unbounded Dirichlet problem provided the density $v$ is the solution of the boundary integral equation

$$
g(y)=\int_{\Gamma} K_{0}(|x-y|) v(x) d s_{y}, \quad y \in \Gamma
$$

which is the first kind boundary integral equation with weakly singular kernel. Assume that $C_{\Gamma}$ denotes the logarithmic capacity of $\Gamma$, when $C_{\Gamma} \neq 1$, the solution of (7) exists and is unique. Once the solution $v(x)$ is solved by (7), the value $u(x)\left(x \in R^{2} \backslash \Gamma\right)$ can be got by (2).

Boundary element method (BEM) [1, 3], finite element method (FEM) [4], and some meshless methods $[5,6]$ have been developed for numerical solutions of the Helmholtz equation. Huang and Wang [7] considered numerical solutions of the boundary integral equation of the Helmholtz equation with mixed boundary value problem on a smooth closed curve by the mechanical quadrature method [8] and extrapolation algorithm. In this case, the solution of the equation is smooth. The boundary element-free method was proposed by Chen, $\mathrm{Liu}$, and $\mathrm{Li}$ [2] for solving two-dimensional exterior and interior Helmholtz equations with mixed boundary value problem. The large wave numbers Helmholtz equation was solved by Wang, Zhai, and Zhang [9] using a new weak Galerkin mixed finite element method. Dastour and Liao [10] presented the finite difference method for solving the two-dimensional Helmholtz problems. A radial basis function-generated finite difference scheme was introduced by London̄o and Montegranario [11] to get the numerical solutions of the two-dimensional Helmholtz equation. Celiker and Lin [12] solved the Helmholtz equation with the Dirichlet boundary value problem by using a conforming finite element method and obtained the high precision numerical solutions, etc. In the above numerical methods, the boundary element method is a good choice due to the reduction of the dimension of boundary value problems. Boundary element method can be 
used to transform boundary value problems into boundary integral equations which include singular integral [1]. There are numerical methods to solve the boundary integral equations (7), such as Galerkin method [13], collocation method [14], and meshless methods [15]. However, these methods are either of low accuracy or complicated or unstable.

The focus of the paper is to investigate the numerical solutions of the Helmholtz equation with Dirichlet problems for the smooth open arc by using a high-accuracy quadrature method which has the characteristics of high-accuracy, low computational complexity, and good stability. We firstly convert the problem into a weakly singular boundary integral equation by using the potential theory. Because the boundary integral equation is defined on the smooth open arc, its solution is singular at the end of the open arc, which seriously affects the accuracy of the numerical solutions. In order to obtain high accuracy numerical solutions, we use Sidi transform to eliminate the singularity of solution at the end of open arc. Further, we construct a high-accuracy quadrature method according to the specific quadrature formula and discrete the equation to get a system of linear equations. Then, we prove the convergence of the numerical solutions and the stability of the method, and prove that the error has a single parameter asymptotic expansion with odd power $O\left(h^{3}\right)$, and the discrete matrix has a good condition number. The higher accuracy can be obtained by the EA. In the end, numerical results support our theoretical analysis.

The remainder of the paper is organized as follows. We analyze the singularities of the solutions and integral kernels in Sect. 2. A quadrature method with high-accuracy is constructed to discrete the integral equation by using singular integral formula in Sect. 3. Furthermore, we prove the convergence and stability of the method. In Sect. 4, we get the single parameter asymptotic expansion of the error and construct the EA based on the expansion to improve the accuracy of the numerical solutions. A numerical example is given in Sect. 5 to verify our theoretical analysis.

\section{Singularity analysis of integral kernels and solutions}

For the convenience of discussion, we assume that the boundary $\Gamma$ is described by the parameter mapping: $x=x(t)=\left(x_{1}(t), x_{2}(t)\right), t \in[0,1]$ with $x(t) \in C^{m}[0,1], m \in N$ and also assume $y=x(s)=\left(x_{1}(s), x_{2}(s)\right), s \in[0,1]$. Under the parameter mapping, (7) can be converted into the following integral equation:

$$
g(s)=\int_{0}^{1} K_{0}(|x(t)-x(s)|) v(x(t))\left|x^{\prime}(t)\right| d t, \quad s \in[0,1]
$$

with $g(s)=g(x(s))$. Since the function $v$ is singular at $t=0$ and $t=1$, we use Sidi transformation [16] to eliminate singularity, which is defined as follows:

$$
t=\psi_{p}(\tau):[0,1] \rightarrow[0,1], \quad p \in N
$$

where $\psi_{p}(\tau)=\vartheta_{p}(\tau) / \vartheta_{p}(1), \vartheta_{p}(\tau)=\int_{0}^{\tau}(\sin (\pi \rho))^{p} d \rho$. Hence (8) is equivalent to

$$
g(s)=\int_{0}^{1} K_{0}(|x(\tau)-x(s)|) \nu(\tau)\left|x^{\prime}\left(\psi_{p}(\tau)\right)\right| \psi_{p}^{\prime}(\tau) d \tau, \quad s \in[0,1],
$$

where $x(\tau)=x\left(\psi_{p}(\tau)\right), v(\tau)=v\left(x\left(\psi_{p}(\tau)\right)\right)$, and $\psi_{p}^{\prime}(\tau)$ has zero points with degree $p$ at $\tau=0$ and $\tau=1$. 
Some integral operators on $[0,1]$ are defined as follows:

$$
(T w)(s)=\int_{0}^{1} t(s, \tau) w(\tau) d \tau
$$

with $t(s, \tau)=-\frac{1}{2 \pi} \ln \left|2 e^{-1 / 2} \sin (\pi(s-\tau))\right|, w(\tau)=v(\tau)\left|x^{\prime}\left(\psi_{p}(\tau)\right)\right| \psi_{p}^{\prime}(\tau)$,

$$
\left(K_{1} w\right)(s)=\int_{0}^{1} k_{1}(s, \tau) w(\tau) d \tau
$$

with $k_{1}(s, \tau)=-\frac{1}{2 \pi}\left[\ln |x(s)-x(\tau)|-\ln \left|2 e^{-1 / 2} \sin (\pi(s-\tau))\right|\right]$, and

$$
\left(K_{2} w\right)(s)=\int_{0}^{1} k_{2}(s, \tau) w(\tau) d \tau
$$

with $k_{2}(s, \tau)=k_{2}(x(s), x(\tau))$. From (11), (12), and (13), we transform (10) into the following matrix operator equation:

$$
\left(T+K_{1}+K_{2}\right) w=\tilde{G},
$$

where $\tilde{G}=g(s)$ and $w=w(\tau)$ with $w(0)=w(1)=0$ if $p$ is sufficiently large. The Symm operator $T$ is an isomorphism between $H^{l}[0,1]$ and $H^{l+1}[0,1]$ for any real $l$, where $H^{l}[0,1]$ is the periodic Sobolev space with period 1, so (14) is equivalent to

$$
\left(E+T^{-1}\left(K_{1}+K_{2}\right)\right) w=G
$$

where $E$ is the identity operator and $G=T^{-1} \tilde{G}$.

Next, we analyze the singularity of the solution $v$ of (7), Let $Q_{1}$ and $Q_{2}$ be the end of $\Gamma$, and assume the endpoints correspond to the parameter values $t_{m}(m=1,2)$. It is well known that the solution $v$ has singularity at points $t_{m}(m=1,2)[17,18]$. The solution $v$ can be described as the following form:

$$
v(x(t))=t^{\beta_{m-1}}(1-t)^{\beta_{m}} g(t)
$$

where $g(0) \neq 0, g(1) \neq 0$, and $\beta_{m} \geq-\frac{1}{2}$. Further, we get

$$
w(\tau)=d_{1} g(0) \tau^{(p+1) \beta_{m-1}+p}\left(1+O\left(\tau^{2}\right)\right), \quad \tau \rightarrow 0^{+}
$$

and

$$
w(\tau)=d_{2} g(1)(1-\tau)^{(p+1) \beta_{m}+p}\left(1+O\left((1-\tau)^{2}\right)\right), \quad \tau \rightarrow 1^{-},
$$

where $d_{1}$ and $d_{2}$ are constants. From (17) and (18), it is easy to know that $w(\tau)$ is bounded in $[0,1]$ and has zeros at $\tau=0$ and $\tau=1$.

Then we get the following results hold:

(1) $t(s, \tau)$ is a logarithmic singular function on $s \in[0,1]$ and $\tau \in[0,1]$;

(2) $k_{1}(s, \tau)$ is a continuous function on $s \in[0,1]$ and $\tau \in[0,1]$;

(3) $k_{2}(s, \tau)$ is a continuous function on $s \in[0,1]$ and $\tau \in[0,1]$;

(4) The solution $w(\tau)$ is a smooth function. 


\section{High-accuracy quadrature method}

Lemma 1 ([16]) Assume $g(x)$ and $h(x) \in C^{2 m}[a, b] . G(x)=\ln |x-t| g(x)+h(x)$ is a periodic function with a period of $b-a$ and at least $2 m$ differentiable in $\mathrm{t}=\{-\infty,+\infty\} \backslash\{t+$ $k T\}_{\{k=-\infty\}}^{\{+\infty\}}$. There exists a quadrature formula as follows:

$$
Q_{n}(G)=h \sum_{j=1, x_{j} \neq t} G\left(x_{j}\right)+h(t) h+\ln \left(\frac{h}{2 \pi}\right) g(t) h
$$

and the error is given by

$$
E_{n}(G)=\int_{a}^{b} G(x) d x-Q_{n}(G)=2 \sum_{j=1}^{m-1} \frac{\zeta^{\prime}(-2 j)}{(2 j) !} g^{(2 j)}(t) h^{2 j+1}+O\left(h^{2 m}\right),
$$

where $\zeta^{\prime}(t)$ is the derivative of the Riemann zeta function and $h$ is the mesh widths.

Let us assume that $h=1 / n(n \in N)$ denotes mesh widths, and $s_{j}=\tau_{j}=(j-1 / 2) h, j=$ $1, \ldots, n$, are middle nodes. For an integral operator $M$ with continuous kernel $m(s, \tau)$ as $K_{1}$ and $K_{2}$, by the midpoint or the trapezoidal rule [19], the Nyström approximation $M^{h}$ is defined

$$
\left(M^{h} w\right)(s)=h \sum_{j=1}^{n} m\left(s, \tau_{j}\right) w\left(\tau_{j}\right), \quad s \in[0,1]
$$

with the error bounds

$$
(M w)(s)-\left(M^{h} w\right)(s)=O\left(h_{m}^{2 l}\right) .
$$

For the logarithmic singular operator $T$, by the quadrature formula (19), the Fredholm approximation $T^{h}$ is defined

$$
\left(T^{h} w\right)\left(s_{i}\right)=\frac{h}{2 \pi} \sum_{j=1, s_{i} \neq \tau_{j}}^{n} \ln \left|2 e^{-1 / 2} \sin \pi\left(s-\tau_{j}\right)\right| w\left(\tau_{j}\right)+\frac{h}{2 \pi} \ln \left|\frac{2 \pi e^{-1 / 2} h}{2 \pi}\right| w\left(s_{i}\right)
$$

with the error bounds

$$
(T w)\left(s_{i}\right)-\left(T^{h} w\right)\left(s_{i}\right)=-\frac{2}{\pi} \sum_{\mu=1}^{2 l-1} \frac{\zeta^{\prime}(-2 \mu)}{(2 \mu) !}\left[w\left(s_{i}\right)\right]^{2 \mu} h_{m}^{2 \mu+1}+O\left(h_{m}^{2 l}\right), \quad i=1, \ldots, n .
$$

From (21) and (23), we can get the approximate equation of (14) as follows:

$$
\left(T^{h}+K_{1}^{h}+K_{2}^{h}\right) w^{h}=\tilde{G}^{h}
$$

where $w^{h}=\left(w\left(\tau_{1}\right), \ldots, w\left(\tau_{n}\right)\right)$ and $\tilde{G}^{h}=\left(\tilde{G}\left(\tau_{1}\right), \ldots, \tilde{G}\left(\tau_{n}\right)\right)$. It is obvious that (25) is a linear equation with $n$ unknowns. The solution $w^{h}$ is solved by (25), then the value $u(y)(y \in$ $\left.R^{2} \backslash \Gamma\right)$ can be got by the following form:

$$
u^{h}(y)=h \sum_{j=1}^{n} K_{0}\left(\left|y-x\left(\tau_{j}\right)\right|\right) w\left(\tau_{j}\right) .
$$


To discuss a unique solution existing for (25), we firstly obtain that the operator $T^{h}$ is invertible. From (23) we know that $T^{h}$ is the circular matrix, which is expressed by

$$
T^{h}=\text { circulate }\left(-\frac{h}{2 \pi} \ln \left|e^{-1 / 2} h\right|, \ldots,-\frac{h}{2 \pi} \ln \left|2 e^{-1 / 2} \sin (\pi(n-1) h)\right|\right) .
$$

Lemma 2 There are two constants $c_{1}>0$ and $c_{2}>0$ independent of $h$, which make the eigenvalues $\lambda_{\alpha}(\alpha=1, \ldots, n)$ of matrix $T^{h}$ satisfy $c_{1} \geq \lambda_{\alpha} \geq c_{2} h$.

Proof Since $\sin (\pi-x)=\sin (x)$, it is easy to see that $T^{h}$ is a symmetric circular matrix. Further, we get $\lambda(\alpha)=F\left(\epsilon^{\alpha}\right) / 2 \pi$ with $\epsilon=e^{2 \pi \sqrt{-1} / n}$ and

$$
F(z)=-h\left\{\ln \left|h e^{-1 / 2}\right|+\sum_{j=1}^{n-1} z^{j} \ln \left|2 e^{-1 / 2} \sin (j \pi / n)\right|\right\} .
$$

To estimate the upper bound, we first prove the following identity:

$$
\prod_{j=1}^{n-1} \sin \left(\frac{j \pi}{n}\right)=\frac{n}{2^{n-1}}
$$

Because the polynomial $x^{n}-1$ can be factorized by its complex roots, we obtain

$$
x^{n}-1=(x-1) \prod_{j=1}^{n-1}\left(x-e^{2 \pi i j / n}\right)
$$

and

$$
1+x+x^{2}+\cdots+x^{n-1} \prod_{j=1}^{n-1}\left(x-e^{2 \pi i j / n}\right)
$$

Let $x=1$, we have

$$
n=\prod_{j=1}^{n-1}\left(1-e^{2 \pi i j / n}\right)=\prod_{j=1}^{n-1}\left|e^{\pi i j n}\right|\left|e^{-\pi i j / n}-e^{\pi i j / n}\right|=2^{n-1} \prod_{j=1}^{n-1} \sin (\pi j / n) .
$$

Based on the above equation, identity (28) can be got. From (28) we have

$$
F(1)=-h\left\{\ln \left|h e^{-1 / 2}\right|+\sum_{j=1}^{n-1} \ln \left|2 e^{-1 / 2} \sin (j \pi / n)\right|\right\}=\frac{1}{2},
$$

and obtain $\lambda_{0}=1 / 4 \pi$.

For $\alpha=1, \ldots, n-1$, we have

$$
\lambda_{\alpha}=-\frac{h}{2 \pi}\left\{\ln \left|h e^{-1 / 2}\right|+\Psi\left(\epsilon^{\alpha}\right)\right\}=\left(\ln (2 n)+(1 / 2-\ln 2) \Phi\left(\epsilon^{\alpha}\right)-\Psi\left(\epsilon^{\alpha}\right)\right) / 2 \pi n
$$

with $\Psi(z)=\sum_{j=1}^{n-1} z^{j} \ln |\sin (j \pi / n)|$ and $\Phi\left(\epsilon^{\alpha}\right)=1+z+\cdots+z^{n-1}$. 
Since $\Psi\left(\epsilon^{\alpha}\right)=n \delta_{\alpha, 0}$ and $(1 / 2-\ln 2)<0$, by $(28)$, we get

$$
|\Psi(z)| \leq \sum_{j=1}^{n-1}-\ln |\sin (j \pi / n)|=-\ln \left(\prod_{j=1}^{n-1} \sin (j \pi / n)\right)=-\ln n+(n-1) \ln 2
$$

and obtain the upper bound of $\lambda_{\alpha}$

$$
\lambda_{\alpha} \leq \frac{1}{2 \pi n}(\ln (2 n)-\ln n+(n-1) \ln 2)=\frac{\ln 2}{2 \pi}
$$

For the lower bound estimate, we first have

$$
\begin{aligned}
\lambda_{\alpha}^{\prime} & =\ln \left|e^{-1 / 2} / n\right|+\sum_{j=1}^{n-1} \cos (2 \alpha j \pi / n) \ln \left|2 e^{-1 / 2} \sin (j \pi / n)\right| \\
& =-\ln n+\sum_{j=1}^{n-1} \cos (2 \alpha \pi / n) \ln |2 \sin (j \pi / n)|,
\end{aligned}
$$

where $\lambda_{\alpha}^{\prime}=-2 \pi n \lambda_{\alpha}$.

From the expansion of the function $[20] \psi(z)=-\gamma-1 / z+z \sum_{j=1}^{\infty} 1 /[j(j+z)]$, we have

$$
\psi(\alpha / n)=\ln 2-\gamma-\ln n-(\pi / 2) \cot (\alpha \pi / n)+\sum_{j=1}^{n-1} \cos (2 \alpha \pi / n) \ln |2 \sin (j \pi / n)|
$$

and get

$$
\begin{aligned}
\sum_{j=1}^{n-1} \cos (2 \alpha \pi / n) \ln |2 \sin (j \pi / n)|= & -\ln 2+\ln n+(\pi / 2) \cot (\alpha \pi / n) \\
& -n / \alpha+\alpha / n \sum_{j=1}^{\infty} \frac{1}{j(j+\alpha / n)} .
\end{aligned}
$$

Further, we obtain

$$
\lambda_{\alpha}^{\prime}=-\ln 2+(\pi / 2) \cot (\alpha \pi / n)-n / \alpha+\alpha / n \sum_{j=1}^{\infty} \frac{1}{j(j+\alpha / n)} .
$$

Substituting

$$
\cot (\alpha \pi / n)=n / \alpha \pi-\alpha \pi /(3 n)-\cdots-2^{2 j}\left|B_{2 j}\right| /(2 j) !(\alpha \pi / n)^{2 j-1}-\cdots
$$

into (30) yields

$$
\begin{aligned}
\lambda_{\alpha}^{\prime}= & -\ln 2+\left(n /(2 \alpha)-\alpha \pi^{2} /(6 n)-\cdots-2^{2 j}\left|B_{2 j}\right| /(2 j) !(\alpha \pi / n)^{2 j-1} \pi-\cdots\right) \\
& -n / \alpha+\alpha / n \sum_{j=1}^{\infty} \frac{1}{j(j+\alpha / n)},
\end{aligned}
$$


and

$$
\begin{aligned}
\lambda_{\alpha}= & \ln 2 /(2 \pi n)+\left\{\left(1 /(2 \alpha)+\alpha \pi^{2} /\left(6 n^{2}\right)+\cdots-2^{2 j}\left|B_{2 j}\right| /(2 j) !(\alpha \pi / n)^{2 j-1} \pi / n+\cdots\right)\right. \\
& \left.-\alpha / n^{2} \sum_{j=1}^{\infty} \frac{1}{j(j+\alpha / n)}\right\} /(2 \pi),
\end{aligned}
$$

where $B_{j}$ is the Bernoulli number. Further, from

$$
\alpha \pi^{2} /\left(6 n^{2}\right)-\alpha / n^{2} \sum_{j=1}^{\infty} \frac{1}{j(j+\alpha / n)}>\alpha / n^{2}\left\{\sum_{j=1}^{\infty}\left[j^{-2}-\frac{1}{j(j+\alpha / n)}\right]\right\}>0,
$$

we obtain

$$
\lambda_{\alpha}>1 /(4 \pi \alpha)+(\alpha \pi) /\left(12 n^{2}\right)+\cdots>1 /(4 \pi \alpha)>1 /(4 \pi n) .
$$

From (29) and (31), we complete the proof of Lemma 2.

Corollary 1 (1) The condition number of matrix $T^{h}$ is $O(n)$; (2) the matrix $T^{h}$ is invertible, and its inverse matrix $\left(T^{h}\right)^{-1}$ is uniformly bounded, and the spectral norm satisfies $\left\|\left(T^{h}\right)^{-1}\right\|=O(n)$.

Based on the above corollary, (25) is equivalent to

$$
\left(E^{h}+\left(T^{h}\right)^{-1}\left(K_{1}^{h}+K_{2}^{h}\right)\right) w^{h}=\left(T^{h}\right)^{-1} G^{h} .
$$

In the following, we will discuss the convergence of the numerical solutions of (32). First, some special operators and subspaces are introduced.

A subspace $C_{0}[0,1] \subset C[0,1]$ is defined as

$$
C_{0}[0,1]=\left\{v(t) \in C[0,1]: v(t) / \sin ^{3}(\pi t) \in C[0,1]\right\}
$$

with a norm $\|v\|^{*}=\max _{0 \leq t \leq 1}\left|v(t) / \sin ^{3}(\pi t)\right|$.

A piecewise linear function subspace is defined as

$$
S^{h}=\operatorname{span}\left\{e_{j}(t), j=1, \ldots, n\right\} \subset C_{0}[0,1]
$$

where the basis function $e_{j}(t)$ satisfies $e_{j}\left(t_{i}\right)=\delta_{j i}$ and $\left\{t_{i}\right\}_{i=0}^{n_{m}-1}$ denotes the basis points. A prolongation operator is defined as $L^{h}: R^{n} \rightarrow S^{h}$ and it satisfies

$$
L^{h} v=\sum_{j=1}^{n} v_{j} e_{j}(t), \quad \forall v=\left(v_{1}, \ldots, v_{n}\right) \in R^{n}
$$

A restricted operator is defined as $F^{h}: C_{0}[0,1] \rightarrow R^{n}$ and it satisfies

$$
F^{h} v=\left(v\left(t_{1}\right), \ldots, v\left(t_{n}\right)\right) \in R^{n}, \quad \forall v \in C_{0}[0,1]
$$

Next, we provide some lemmas to prove the convergence. 
Lemma $3\left\{L^{h}\left(T^{h}\right)^{-1} F^{h} T: C^{3}[0,1] \rightarrow C[0,1]\right\}$ is convergent to $E$.

Proof From (23), assume that $\forall \psi \in C^{3}[0,1]$ and $\psi^{h}$ are the solutions of auxiliary equations $T \psi=\rho$ and $T^{h} \psi^{h}=F^{h} \rho$, respectively. We get

$$
T \psi\left(s_{i}\right)=T^{h} \psi\left(s_{i}\right)+\eta_{i}
$$

in which $\eta_{i}=O\left(h^{3}\right)(i=1, \ldots, n)$. Denoting $e\left(s_{i}\right)=\psi^{h}\left(s_{i}\right)-\psi\left(s_{i}\right)$, we get

$$
T^{h} e\left(s_{i}\right)=\left.T^{h}\left(\psi^{h}-\psi\right)\right|_{s=s_{i}}=\left.F^{h} \rho\right|_{s=s_{i}}-\left(T \psi\left(s_{i}\right)-\eta_{i}\right)=\eta_{i}=O\left(h^{3}\right)
$$

and

$$
T^{h} e=\eta, \quad e^{\prime}=\left(e\left(s_{1}\right), \ldots, e\left(s_{n}\right)\right), \quad \eta^{\prime}=\left(\eta_{1}, \ldots, \eta_{n}\right) .
$$

We get $e=\left(T^{h}\right)^{-1} \eta$ with $\|e\|=O\left(h^{2}\right)$ by Corollary 1 , and

$$
\begin{aligned}
\|e\| & =\left\|\left(T^{h}\right)^{-1} \varepsilon\right\|=\left\|F^{h} T^{-1} \rho-\left(T^{h}\right)^{-1} F^{h} \rho\right\| \\
& =\left\|F^{h} \phi-\left(T^{h}\right)^{-1} F^{h} T \phi\right\| .
\end{aligned}
$$

By $L^{h} F^{h} \rightarrow E$ as $h \rightarrow 0$ in $£\left(C^{3}[0,1] \rightarrow C[0,1]\right)$, the proof of Lemma 3 is completed.

Lemma 4 Assume that $T \in £\left(C[0,1], C^{k}[0,1]\right), T^{-1}$ exist, $M \in £\left(C[0,1], C^{k+1}[0,1]\right)$, and the kernel $m(s, \tau)$ of $M$ satisfies $T^{-1} m(s, \tau)=\tilde{m}(s, \tau)$, in which $\tilde{m}(s, \tau)$ is the kernel of $\tilde{M}$. Let $\tilde{m}(s, \tau)$ and $\frac{d}{d s} \tilde{m}(s, \tau)$ be continuous on $[0,1]^{2}$. We have

$$
\left(\tilde{M}^{h} w\right)(s)=h \sum_{j=1}^{n} \tilde{m}\left(s, \tau_{j}\right) w\left(\tau_{j}\right), \quad w \in[0,1]
$$

is the collectively compact convergent to $\tilde{M}$ :

$$
T^{-1} M^{h} \stackrel{c . c}{\rightarrow} T^{-1} M \in £\left(C^{0}[0,1], C^{1}[0,1]\right) .
$$

Proof According to (35), we get

$$
T^{-1} M^{h} w=h \sum_{j=1}^{n} \tilde{m}\left(s, \tau_{j}\right) w\left(\tau_{j}\right) \quad \rightarrow \quad \int_{0}^{1} \tilde{m}(s, \tau) w(\tau) d \tau=T^{-1} M u .
$$

$d(s, \tau)$ is a continuous function on $[0,1]^{2}$, hence we get [21]

$$
T^{-1} M^{h} \stackrel{c . c}{\rightarrow} T^{-1} M \in £\left(C^{0}[0,1], C^{\infty}[0,1]\right)
$$

and

$$
\frac{d}{d s} T^{-1} M^{h} w=\frac{d}{d s}\left[h \sum_{j=1}^{n} \tilde{m}\left(s, \tau_{j}\right) w\left(\tau_{j}\right)\right] .
$$

Since $\frac{d}{d s} \tilde{d}(s, \tau)$ is a continuous function on $[0,1]^{2},(36)$ can be obtained. 
Further the following corollary can be got.

Corollary 2 Let the Nyström approximation $M^{h}$ of $M$ be defined by (21), we have

$$
L^{h}\left(T^{h}\right)^{-1} F^{h} M^{h} \stackrel{c . c}{\rightarrow} T^{-1} M \in C[0,1] \rightarrow C[0,1] .
$$

Proof Under the parameter transformations (9), the kernel $m(s, \tau)$ of $M$ is continuous, and its high order derivatives are also continuous. From

$$
L^{h}\left(T^{h}\right)^{-1} F^{h} M^{h}=\left(L^{h}\left(T^{h}\right)^{-1} F^{h} T\right)\left(T^{-1} M^{h}\right),
$$

we get

$$
\left\|L^{h}\left(T^{h}\right)^{-1} F^{h} M^{h}\right\|_{0,0} \leq\left\|\left(L^{h}\left(T^{h}\right)^{-1} F^{h} T\right)\right\|_{0,3}\left\|T^{-1} M^{h}\right\|_{3,0},
$$

in which $\|\cdot\|_{m_{2}, m_{1}}$ is the norm of $£\left(C^{m_{1}}[0,1], C^{m_{2}}[0,1]\right)$. By Lemma 4 and $T^{-1} M^{h} \in$ $C[0,1] \rightarrow C^{3}[0,1]$, there exists a constant $c$ independent of $h$ so that

$$
\left\|L^{h}\left(T^{h}\right)^{-1} F^{h} T\right\|_{0,3} \leq c \quad \text { and } \quad\left\|T^{-1} M^{h}\right\|_{3,0} \leq c .
$$

Also using the results of [21] and Lemma 3, we conclude that $\left\{T^{-1} M^{h}: C[0,1] \rightarrow C^{3}[0,1]\right\}$ must be collectively compact convergent to $T^{-1} M$. Hence, the proof is completed.

Let $\left(\hat{T}^{h}\right)^{-1}\left(\hat{K}_{1}^{h}+\hat{K}_{2}^{h}\right)=L^{h}\left(T^{h}\right)^{-1} F^{h}\left(K_{1}^{h}+K_{2}^{h}\right), \hat{w}^{h}=L^{h} w^{h}$, and $\hat{G}=L^{h} G^{h}$. We construct an operator equation

$$
\left(E^{h}+\left(\hat{T}^{h}\right)^{-1}\left(\hat{K}_{1}^{h}+\hat{K}_{2}^{h}\right)\right) \hat{w}^{h}=\left(\hat{T}^{h}\right)^{-1} \hat{G}^{h}
$$

Obviously, if $\hat{w}^{h}$ is the solution of (38), then $F^{h} \hat{w}^{h}$ must be the solution of (32); conversely, if $w^{h}$ is the solution of (32), then $L^{h} w^{h}$ must be the solution of (38). Below we prove that there exists a unique solution $\hat{w}^{h}$ in (38) such that $\hat{w}^{h}$ converges to $w$.

Finally, the main theorem is proved to complete the proof of convergence.

Theorem $1\left\{L^{h}\left(T^{h}\right)^{-1} F^{h}\left(K_{1}^{h}+K_{2}^{h}\right)\right\}$ is collectively convergent to $T^{-1}\left(K_{1}+K_{2}\right) \in C_{0}[0,1]$ :

$$
L^{h}\left(T^{h}\right)^{-1} F^{h}\left(K_{1}^{h}+K_{2}^{h}\right) \stackrel{c . c}{\rightarrow} T^{-1}\left(K_{1}+K_{2}\right)
$$

Proof Let $\Theta=\{v:\|v\| \leq 1, v \in V\}$ be a unit ball. $H=\left\{h^{1}, h^{2}, \ldots\right\}$ is the grid step sequence and $h^{l} \rightarrow 0$ as $l \rightarrow \infty$. We arbitrarily take a sequence $\left\{Z_{h}\right\}_{h \in H}$ in space $\Theta$ and verify that there exists a convergent subsequence in $\left\{L^{h}\left(T^{h}\right)^{-1} F^{h}\left(K_{1}^{h}+K_{2}^{h}\right) Z_{h}\right\}$. Using the results of Lemma 4 and Corollary 2, and by

$$
L^{h}\left(T^{h}\right)^{-1} F^{h} K_{1}^{h} Z_{h}=L^{h}\left(T^{h}\right)^{-1} F^{h} T^{h}\left[\left(T^{h}\right)^{-1} F^{h} K_{1}^{h}\right] Z_{h},
$$


we get

$$
\begin{aligned}
& \left\|L^{h}\left(T^{h}\right)^{-1} F^{h} M^{h}\right\|_{0,0} \\
& \left.\quad \leq\left\|L^{h}\left(T^{h}\right)^{-1} F^{h} T^{h}\right\|_{0,1} \|\left(T^{h}\right)^{-1} F^{h} D^{h}\right] \|_{1,0} \\
& \left.\quad \leq c \|\left(T^{h}\right)^{-1} F^{h} M^{h}\right] \|_{1,0},
\end{aligned}
$$

where $M^{h}$ stands for $K_{1}^{h}$ and $K_{2}^{h}$. Thus we get

$$
L^{h}\left(T^{h}\right)^{-1} F^{h} M^{h} \stackrel{c . c}{\rightarrow} T^{-1} M
$$

An infinite subsequence is found in $\left\{L^{h}\left(T^{h}\right)^{-1} F^{h} K_{1}^{h} Z_{h}\right\}$ by collectively compact theory [21], which converges as $h \rightarrow 0$. Similarly, $\left\{L^{h}\left(T^{h}\right)^{-1} F^{h} K_{2}^{h} Z_{h}\right\}$ has the same result. Hence, it is proved that there exists an infinite subsequence $H^{(1)} \subset H$ such that $\left\{L^{h}\left(T^{h}\right)^{-1} F^{h}\left(K_{1}^{h}+\right.\right.$ $\left.K_{2}^{h}\right) Z_{h}$ \} converges. Similarly, it can be concluded that there exists an infinite subsequence $H^{(d)} \subset H^{(d-1)} \subset \cdots \subset H^{(1)} \subset H$ such that $\left\{L^{h}\left(T^{h}\right)^{-1} F^{h}\left(K_{1}^{h}+K_{2}^{h}\right) Z_{h}\right\}$ converges. Therefore, $\left\{L^{h}\left(T^{h}\right)^{-1} F^{h}\left(K_{1}^{h}+K_{2}^{h}\right)\right\}$ is a collectively compact convergent sequence, and $L^{h}\left(T^{h}\right)^{-1} F^{h}\left(K_{1}^{h}+K_{2}^{h}\right) \stackrel{P}{\rightarrow} T^{-1}\left(K_{1}+K_{2}\right)$, where $\stackrel{P}{\rightarrow}$ shows the point of convergence. The proof is completed.

Meanwhile, the following corollary is presented to obtain the stability of the method.

Corollary 3 Assume that $K_{1}^{h}, K_{2}^{h}$, and $T^{h}$ are defined by (21) and (23), respectively. The eigenvalues of $\Upsilon^{h}=T^{h}+K_{1}^{h}+K_{2}^{h}$ are $\lambda_{i}(i=1, \ldots, n)$. There is the bound of condition number

$$
\operatorname{Cond}\left(\Upsilon^{h}\right)=\frac{\max _{1 \leq i \leq n}\left|\lambda_{i}\left(\Upsilon^{h}\right)\right|}{\min _{1 \leq i \leq n}\left|\lambda_{i}\left(\Upsilon^{h}\right)\right|}=O\left(h^{-1}\right)
$$

\section{Asymptotic expansion of error and EA}

In what follows, the single parameter asymptotic expansion of error is got to describe the EA.

Theorem 2 There exists a function $\Phi$ independent of h such that the following asymptotic expansion with a single parameter holds at nodes:

$$
w^{h}-w=h^{3} \Phi+o\left(h^{3}\right)
$$

where $w^{h} \in S, w \in C^{3}[0,1]$.

Proof By (22) and (24), there exists the single parameter asymptotic expansion

$$
\left(\tilde{G}^{h}-\tilde{G}\right)=h^{3} L^{h} F^{h} \omega+o\left(h^{3}\right)
$$

in which $\omega=-\zeta^{\prime}(-2) g^{\prime \prime}(s) / \pi$.

Using (14), (22), and (24), we obtain

$$
\left(T^{h}+K_{1}^{h}+K_{2}^{h}\right) F^{h}\left(w^{h}-w\right)=\tilde{G}^{h}-L^{h}\left(T^{h}+K_{1}^{h}+K_{2}^{h}\right) F^{h} w
$$




$$
\begin{aligned}
& =\tilde{G}^{h}-\left[\left(T+K_{1}+K_{2}\right) w-h^{3} L^{h} F^{h} \gamma\right]+o\left(h^{3}\right) \\
& =\left(\tilde{G}^{h}-\tilde{G}\right)+h^{3} L^{h} F^{h} \gamma+o\left(h^{3}\right) \\
& =h^{3} L^{h} F^{h} \eta+o\left(h^{3}\right)
\end{aligned}
$$

where $\gamma=-\zeta^{\prime}(-2) \nu^{\prime \prime}(t) / \pi$, and $\eta=\omega+\gamma$. From Theorem 1 , we get

$$
\left(E+L^{h}\left(T^{h}\right)^{-1} F^{h}\left(K_{1}^{h}+K_{2}^{h}\right)\right)\left(w^{h}-w\right)=h^{3}\left(T^{h}\right)^{-1} L^{h} F^{h} \eta+o\left(h^{3}\right) .
$$

We construct the following auxiliary equation:

$$
\left(E+T^{-1}\left(K_{1}+K_{2}\right)\right) \Phi=T^{-1} \eta
$$

and its approximate equation

$$
\left(E+L^{h}\left(T^{h}\right)^{-1} F^{h}\left(K_{1}^{h}+K_{2}^{h}\right)\right) \Phi^{h}=\left(T^{h}\right)^{-1} L^{h} F^{h} \eta .
$$

By substituting (46) into (44), we get

$$
\left(E+L^{h}\left(T^{h}\right)^{-1} F^{h}\left(K_{1}^{h}+K_{2}^{h}\right)\right)\left(w^{h}-w-h^{3} \Phi^{h}\right)=o\left(h^{3}\right) .
$$

Since $\left(E+L^{h}\left(T^{h}\right)^{-1} F^{h}\left(K_{1}^{h}+K_{2}^{h}\right)\right)$ is uniformly bounded, from Theorem 1 we get

$$
w^{h}-w-h^{3} \Phi^{h}=o\left(h^{3}\right) .
$$

Replacing $\Phi^{h}$ with $\Phi$, we can complete the proof.

Based on the single parameter asymptotic expansion (41), the EA can be constructed as follows [22]:

$$
\hat{u}^{h}\left(s_{j}\right)=\frac{1}{7}\left(8 u^{h / 2}\left(s_{j}\right)-u^{h}\left(s_{j}\right)\right),
$$

with the error estimate $\left\|\hat{u}^{h}\left(s_{j}\right)-u\left(s_{j}\right)\right\|=o\left(h^{3}\right)$.

By the extrapolation results, the following a posteriori estimate can be obtained:

$$
\begin{aligned}
\left\|u^{h / 2}\left(s_{j}\right)-u\left(s_{j}\right)\right\| \leq & \left\|\frac{8}{7} u^{h / 2}\left(s_{j}\right)-\frac{1}{7} u^{h}\left(s_{j}\right)-u\left(s_{j}\right)\right\| \\
& +\frac{1}{7}\left\|u^{h / 2}\left(s_{j}\right)-u^{h}\left(s_{j}\right)\right\| \leq \frac{1}{7}\left\|u^{h / 2}\left(s_{j}\right)-u^{h}\left(s_{j}\right)\right\|+o\left(h^{3}\right) .
\end{aligned}
$$

The self-adaptive algorithm can be got by using the above inequality. Obviously, the EA is not complicated, but the accuracy of the numerical solution is improved.

\section{Numerical example}

In what follows, we provide a numerical example to verify the proposed method. 
Example We consider the scattering of a plane wave $u^{i}$ by a sound-soft cylinder with the smooth open contour cross section with the boundary $\Gamma$, which is described by the parameter mapping $x(t)=\left(2 \sin \frac{t}{2}, \sin t\right), \frac{\pi}{4} \leq t \leq \frac{7 \pi}{4}$. The incident wave is given by $u^{i}(x)=e^{i k d \cdot x}$ with $d$ denoting the unit vector of propagation direction, and the boundary values $g=-u^{i}$ on $\Gamma$ are considered. The far-field pattern $u_{\infty}$ is defined by the asymptotic behavior of the scattered wave

$$
u(y)=\frac{e^{i k|y|}}{\sqrt{|y|}}\left\{u_{\infty}+O(1 /|y|)\right\}, \quad|y| \rightarrow \infty,
$$

uniformly in all directions $\hat{y}=y /|y|$. Based on the following asymptotic formula of the Hankel function

$$
H_{0}^{(1)}(y)=\sqrt{\frac{2}{\pi y}} e^{i(y-\pi / 4)}\{1+O(1 / y)\}, \quad|y| \rightarrow \infty,
$$

the far-field pattern of the single-layer potential (2) is obtained by the form

$$
u_{\infty}(\hat{y})=c \int_{\Gamma} e^{-i k x \cdot y} v(x) d s_{y}
$$

with $c=-\frac{e^{i \pi / 4}}{\sqrt{2 \pi k}}$.

In Tables 1 and 2, we show some approximate values for the far-field patterns $u_{\infty}(d)$ in the forward direction $d=(1,0)$ with $k=1$ and $k=5$, respectively. Let $\operatorname{Re}\left(e_{\infty}(d)\right)$ denote the real part of errors and $\operatorname{Im}\left(e_{\infty}(d)\right)$ denote the imaginary part of errors. Also let $\operatorname{Re}\left(e_{\infty}^{E}(d)\right)$ and $\operatorname{Im}\left(e_{\infty}^{E}(d)\right)$ denote the errors by the EA once. Let ratio $=\frac{\left.e\right|_{n}}{\left.e\right|_{2 n}}$ with $\left.e\right|_{n}$ denote the errors under the mesh widths $h=1 / n$. From Tables 1 and 2 , we can see $\log _{2}$ ratio $\approx 3$, which agrees with Theorem 2 very well, and the EA greatly improves the accuracy of numerical solutions.

In Tables 3 and 4, we show the condition numbers of the discrete matrices with $k=1$ and $k=5$, respectively. Let $\left|\lambda_{\max }\right|$ and $\left|\lambda_{\min }\right|$ denote the absolute values of the maximum eigenvalue and the minimum eigenvalue, respectively. From Tables 3 and 4, we can see $\frac{\operatorname{Cond}_{2 n}}{\left.\operatorname{Cond}\right|_{n}} \approx 2$, which agrees with Corollary 3 very well, where Cond is the condition number.

Table 1 Numerical results for the far-field pattern $u_{\infty}(d)$ with $k=1$

\begin{tabular}{rllllll}
\hline \multicolumn{1}{r}{} & $\operatorname{Re}\left(e_{\infty}(d)\right)$ & ratio & $\operatorname{Re}\left(e_{\infty}^{E}(d)\right)$ & $\operatorname{Im}\left(e_{\infty}(d)\right)$ & ratio & $\operatorname{Im}\left(e_{\infty}^{E}(d)\right)$ \\
\hline 32 & $1.1017 \mathrm{E}-4$ & - & - & $7.3137 \mathrm{E}-5$ & - & - \\
64 & $1.3733 \mathrm{E}-5$ & 8.0220 & $4.3141 \mathrm{E}-8$ & $9.0241 \mathrm{E}-6$ & 8.1046 & $1.3488 \mathrm{E}-7$ \\
128 & $1.7245 \mathrm{E}-6$ & 7.9642 & $8.8248 \mathrm{E}-9$ & $1.1064 \mathrm{E}-6$ & 8.1560 & $2.4662 \mathrm{E}-8$ \\
256 & $2.1803 \mathrm{E}-7$ & 7.9094 & $2.8222 \mathrm{E}-9$ & $1.2934 \mathrm{E}-7$ & 8.5542 & $1.0240 \mathrm{E}-8$ \\
\hline
\end{tabular}

Table 2 Numerical results for the far-field pattern $u_{\infty}(d)$ with $k=5$

\begin{tabular}{rllllll}
\hline$n$ & $\operatorname{Re}\left(e_{\infty}(d)\right)$ & ratio & $\operatorname{Re}\left(e_{\infty}^{E}(d)\right)$ & $\operatorname{Im}\left(e_{\infty}(d)\right)$ & ratio & $\operatorname{Im}\left(e_{\infty}^{E}(d)\right)$ \\
\hline 32 & $2.3166 \mathrm{E}-2$ & - & - & $1.0459 \mathrm{E}-2$ & - & - \\
64 & $2.7547 \mathrm{E}-3$ & 8.4096 & $1.6118 \mathrm{E}-4$ & $1.2172 \mathrm{E}-3$ & 8.5930 & $1.0312 \mathrm{E}-4$ \\
128 & $3.4001 \mathrm{E}-4$ & 8.1017 & $4.9395 \mathrm{E}-6$ & $1.5062 \mathrm{E}-4$ & 8.0808 & $1.7397 \mathrm{E}-6$ \\
256 & $4.1964 \mathrm{E}-5$ & 8.1024 & $6.1389 \mathrm{E}-7$ & $1.8663 \mathrm{E}-5$ & 8.0707 & $1.8853 \mathrm{E}-7$ \\
\hline
\end{tabular}


Table 3 The condition number with $k=1$

\begin{tabular}{lllll}
\hline & $n$ & & & \\
\cline { 2 - 5 } & 32 & 64 & 128 & 256 \\
\hline$\left|\lambda_{\min }\right|$ & $6.9278 \mathrm{E}-3$ & $3.4514 \mathrm{E}-3$ & $1.7242 \mathrm{E}-3$ & $8.6192 \mathrm{E}-4$ \\
$\left|\lambda_{\max }\right|$ & 0.1902 & 0.1903 & 0.1903 & 0.1903 \\
Cond & 27.4476 & 55.1251 & $1.1036 \mathrm{E}+002$ & $2.2078 \mathrm{E}+002$ \\
\hline
\end{tabular}

Table 4 The condition number with $k=5$

\begin{tabular}{lllll}
\hline & $n$ & & \\
\cline { 2 - 5 } & 32 & 64 & 128 & 256 \\
\hline$\left|\lambda_{\min }\right|$ & $7.0456 \mathrm{E}-3$ & $3.4653 \mathrm{E}-3$ & $1.7259 \mathrm{E}-3$ & $8.6213 \mathrm{E}-4$ \\
$\left|\lambda_{\max }\right|$ & 0.1067 & 0.1069 & 0.1069 & 0.1070 \\
Cond & 15.1410 & 30.8440 & 61.9599 & $1.2405 \mathrm{E}+002$ \\
\hline
\end{tabular}

\section{Conclusions}

In this paper, we discuss the high-accuracy quadrature method and the EA for scattering problems for a smooth open arc, there exist the following advantages: (a) Computing an entry of the discrete matrices is straightforward and simple, without any singular integrals, hence the method is appropriate to solve weakly singularity problems; (b) The method possesses the feature of high accuracy. The $O\left(h^{3}\right)$ order accuracy of the error is proved, and the numerical results verify our theoretical analysis. The EA can be used to raise the accuracy of the solutions, see Tables 1 and 2; (c) The condition number of the discrete matrix increases linearly, which shows that the method is stable. Although the method has the characteristics of high accuracy, low computational complexity, and good stability, it also has a limitation, that is, the method is effective for solving weakly singular boundary integral equations, but not for strongly singular and hypersingular boundary integral equations. Based on the research results in $[23,24]$, we intend to apply the method to the Neumann problems for the Helmholtz equation and the exterior acoustic problems with arbitrary and high wave numbers in the future.

Acknowledgements

The authors would like to express sincere thanks to the anonymous referees for valuable comments and suggestions.

Funding

Research of the first author was supported by the Program of Chengdu Normal University (YJRC2018-1; CS18ZDZ02) and research of the third author was supported by the National Natural Science Foundation of China $(11661005 ; 11301070)$.

Availability of data and materials

Data sharing not applicable to this article as no datasets were generated or analyzed during the current study.

Ethics approval and consent to participate

Not applicable.

Competing interests

The authors declare that they have no competing interests.

Consent for publication

Not applicable.

Authors' contributions

The authors declare that the study was realized in collaboration with equal responsibility. All authors read and approved the final manuscript.

\section{Author details}

'School of Mathematics, Chengdu Normal University, Chengdu 611130, China. ${ }^{2}$ School of Mathematical Sciences, University of Electronic Science and Technology of China, Chengdu 611731, China. ${ }^{3}$ School of Sciences, East China University of Technology, Nanchang 330013, China. 


\section{Publisher's Note}

Springer Nature remains neutral with regard to jurisdictional claims in published maps and institutional affiliations.

Received: 16 April 2020 Accepted: 30 July 2020 Published online: 06 August 2020

\section{References}

1. Kirkup, S., Wu, S.F.: The Boundary Element Method in Acoustics. Cambridge University Press, Cambridge (2007)

2. Chen, L.C., Liu, X., Li, X.L.: The boundary element-free method for 2D interior and exterior Helmholtz problems. Comput. Math. Appl. 77(3), 846-864 (2019)

3. Bergman, D.R.: Boundary element method. In: Computational Acoustics: Theory and Implementation (2018)

4. Chen, S.H.: Adaptive techniques in the finite element method. In: Computational Geomechanics and Hydraulic Structures (2019)

5. Jin, B., Zheng, Y.: A meshless method for some inverse problems associated with the Helmholtz equation. Comput. Methods Appl. Mech. Eng. 195(22), 2270-2288 (2006)

6. Tadeu, A., Chen, C.S., António, J., et al.: A boundary meshless method for solving heat transfer problems using the Fourier transform. Adv. Appl. Math. Mech. 3(5), 572-585 (2011)

7. Huang, J., Wang, Z.: Extrapolation algorithms for solving mixed boundary integral equations of the Helmholtz equation by mechanical quadrature methods. SIAM J. Sci. Comput. 31(6), 4115-4129 (2009)

8. Li, H., Huang, J.: Mechanical quadrature method and splitting extrapolation algorithm for the boundary integral equation of the axisymmetric anisotropic Darcy's equation. Int. J. Comput. Math. 94(1), 135-150 (2017)

9. Wang, R.S., Wang, X.S., Zhai, Q.L., et al.: A weak Galerkin mixed finite element method for the Helmholtz equation with large wave numbers. Numer. Methods Partial Differ. Equ. 34(3), 1009-1032 (2018)

10. Dastour, H., Liao, W.Y.: A fourth-order optimal finite difference scheme for the Helmholtz equation with PML. Comput. Math. Appl. 78(6), 2147-2165 (2019)

11. Londoño, M.A., Montegranario, H.: Radial basis function-generated finite differences with Bessel weights for the $2 \mathrm{D}$ Helmholtz equation. Eng. Anal. Bound. Elem. 105, 257-266 (2019)

12. Celiker, E., Lin, P.: A highly-accurate finite element method with exponentially compressed meshes for the solution of the Dirichlet problem of the generalized Helmholtz equation with corner singularities. J. Comput. Appl. Math. 361(1), 227-235 (2019)

13. Wang, B., Wang, R., Xu, Y.S.: Fast Fourier-Galerkin methods for first-kind logarithmic-kernel integral equations on open arcs. Sci. China Math. 53(1), 1-22 (2010)

14. Chen, X.L., Xie, Z.Q., Luo, J.S.: A wavelet collocation method for boundary integral equations of the modified Helmholtz equation. Appl. Math. Comput. 321(15), 300-312 (2018)

15. Assari, P., Dehghan, M.: Solving a class of nonlinear boundary integral equations based on the meshless local discrete Galerkin (MLDG) method. Appl. Numer. Math. 123, 137-158 (2018)

16. Sidi, A., Israrli, M.: Quadrature methods for periodic singular Fredholm integral equation. J. Sci. Comput. 3, 201-231 (1988)

17. Yan, Y., Sloan, l.: On integral equation of the first kind with logarithmic kernels. J. Integral Equ. Appl. 1, 1-31 (1988)

18. Sloan, I.H., Spence, A.: The Galerkin method for integral equations of the first-kind with logarithmic kernel: theory. IMA J. Numer. Anal. 3, 105-122 (1988)

19. Davis, P., Rabinowitz, P.: Methods of Numerical Integration, 2nd edn. Academic Press, New York (1984)

20. Gradshteyan, I.S., Ryzhik, Z.M.: Tables of Integrals, Series and Products. Academic Press, New York (1980)

21. Anselone, P.M.: Collectively Compact Operator Approximation Theory. Prentice Hall, Englewood Cliffs (1971)

22. Liem, C.B., Lü, T., Shih, T.M.: The Splitting Extrapolation Method. World Scientific, Singapore (1995)

23. Chen, L.C., Li, X.L.: A complex variable boundary element-free method for the Helmholtz equation using regularized combined field integral equations. Appl. Math. Lett. 101, 106067 (2020)

24. Chen, L.C., Li, X.L.: Boundary element-free methods for exterior acoustic problems with arbitrary and high wavenumbers. Appl. Math. Model. 72, 85-103 (2019)

\section{Submit your manuscript to a SpringerOpen ${ }^{\mathcal{O}}$ journal and benefit from:}

- Convenient online submission

- Rigorous peer review

- Open access: articles freely available online

- High visibility within the field

- Retaining the copyright to your article

Submit your next manuscript at $\gg$ springeropen.com 